\title{
Experimental Verification of the Blob Model and Delayed Formation of Ps
}

\author{
C. Dauwe, B. Van Waeyenberge and J. De Baerdemaeker \\ Dpt. Subatomic and Radiation Physics, Ghent University \\ Proeftuinstraat 86, 9000, Gent, Belgium
}

\begin{abstract}
Stepanov and Byakov's blob model for the formation of positronium can be considered as an extension of the spur model. However, it offers many extra possibilities and is more sound from the radiation chemistry point of view. A full coding of this model leads to non-exponential lifetime spectra, and standard multi-component analysis is no longer adequate for materials such as polymers. Age momentum correlation experiments were performed on linear PMMA at different temperatures and were analyzed with the non-exponential blob model. We show the adequacy of this model, and moreover an alternative explanation for the young-age broadening and delayed formation of positronium.
\end{abstract}

PACS numbers: 36.10.Dr, 78.70.Bj, 61.80.Fe, 82.35.Lr

\section{Introduction}

Commonly lifetime (LT) spectra in polymers are analyzed as a composition of three exponential decay modes or components, indicated by their lifetimes $\tau_{1,2,3}$ and their intensities $I_{1,2,3}$. The shortest component is associated to the annihilation of para-positronium ( $p$-Ps), the second component to free positrons and the longest one to the ortho-positronium (o-Ps) component. The intensity of the $p$-Ps component should be equal to one third of the intensity of the o-Ps component. Such an interpretation is satisfactory if one is only interested in the o-Ps component and in the relation between $\tau_{o-P s}$ and the mean size of the free volume sites. Detailed analysis leads to a number of discrepancies, which we have discussed in detail in a former paper [1].

In order to solve these discrepancies it is necessary to establish a detailed model of positronium formation, particularly its evolution in time at the sub-nanosecond scale, and to critically test this model to the age momen- 
tum correlation (AMOC) experiments. The blob model for Ps formation was introduced by Byakov and Stepanov [2, 3]. These authors concentrated on calculating the intensity of the long component, particularly its dependence on an applied electrical field, but they ignored the details of the sub-nanosecond behavior of positrons and the age-dependent formation of Ps. Therefore in this paper we will give a short account on recent interpretations of AMOC experiments on the polymer PMMA, and show some new model simulations that may be verified experimentally.

\section{Experimental}

Experimental AMOC data were obtained at the Stuttgart pelletron positron beam $[4,5]$ on linear and cross-linked PMMA specimen at temperatures below and above the glass transition. At each temperature 30 or more consecutive AMOC data sets were taken of 1 hour measuring time each. The individual AMOC data were added up after we checked that the center of mass of the lifetime spectra did not change with time, indicating that there were no positron beam irradiation induced effects. Each summed set contains at least $2 \times 10^{7}$ counts. Summation of the data over the energy axis generates the overall experimental lifetime spectrum $\Lambda_{\exp }(t)$ and the line shape curve $S_{\exp }(t)$ is obtained by calculating the line shape parameter for each time channel.

\section{Data analysis}

In the "white" positron blob model, a positron of several hundred keV loses most of its energy within $10^{-11} \mathrm{~s}$ through ionizing collisions until its energy drops below the ionization threshold. In the final ionizing regime, with the positron energy between $0.5 \mathrm{keV}$ and the ionization threshold, approximately 30 electronion pairs are generated within a spherical volume, called the blob. The subionizing positron further undergoes positron-phonon scattering and may diffuse out of the blob, until it becomes thermalized in a spherical volume slightly bigger than the blob volume. Thus at zero time we consider that:

1) a number of free electrons $n_{\mathrm{b}}$ are radially distributed around the blob center as a Gauss function $G(r)$ with dispersion $\sigma_{\mathrm{b}}$, so the initial electron density is $\rho_{\mathrm{b}}(r, t=0)=n_{\mathrm{b}}(t=0) G(r)$ and $\int 4 \pi r^{2} G(r) \mathrm{d} r=1$;

2 ) the initial positron density $\rho_{+}(r, t=0)$ is also a Gauss function with dispersion $\sigma_{+}>\sigma_{\mathrm{b}}$, and

3 ) only positrons are present, positronium has still to be formed; $\rho_{p-\mathrm{Ps}}(r, t=0)=$ $\rho_{o-\mathrm{Ps}}(r, t=0)=0$.

Blob electrons recombine with the positive ions. We consider that the blob electron distribution $\sigma_{\mathrm{b}}$ remains Gaussian at all times, but that the total number of electrons decreases in an exponential way such that $n_{\mathrm{b}}(t)=n_{\mathrm{b}}(0) \exp \left(-t / \tau_{\mathrm{b}}\right)$, 
where $\tau_{\mathrm{b}}$ is the blob recombination lifetime, which is in the order of $20-30$ ps. The free positrons undergo spatial redistribution through diffusion, and its decay is branched into direct annihilation with a decay constant $\lambda_{+}$and formation of positronium. The formation of positronium at time $t$, at position $r$ is proportional to the overlap of the electron and the positron density or $\left(\mathrm{d} \rho_{\mathrm{Ps}} / \mathrm{d} t\right)_{\text {form }}=\kappa \rho_{\mathrm{e}} \rho_{+}$, where $\kappa$ is the chemical reaction constant for Ps formation. This Ps formation may occur with blob electrons, with shallow trapped electrons, or upon passage through a free-volume hole (i.e. the hole model). $p$-Ps and $o$-Ps are formed with the fixed ratio of 1 to 3 . These processes are described by a set of rate equations

$$
\left\{\begin{array}{l}
\dot{\rho}_{p-\mathrm{Ps}}=(1 / 4) \sum \kappa_{i} \rho_{i} \rho_{+}-\lambda_{p-\mathrm{Ps}} \rho_{p-\mathrm{Ps}} \\
\dot{\rho}_{o-\mathrm{Ps}}=(3 / 4) \sum \kappa_{i} \rho_{i} \rho_{+}-\lambda_{o-\mathrm{Ps}} \rho_{o-\mathrm{Ps}} \\
\dot{\rho}_{+}=-\left(\sum \kappa_{i} \rho_{i}+\lambda_{+}\right) \rho_{+}+D_{+} \nabla^{2} \rho_{+}
\end{array}\right.
$$

where the index $i$ indicates all the Ps-forming channels. The particle densities are space and age dependent i.e. $\rho_{i}=\rho_{i}(r, t)$ but the shallow trapped electrons and the free volume holes are supposed uniformly distributed over space and constant with positron age. The resulting lifetime spectrum $\Lambda(t)$ and line shape curve $S(t)$ are

$$
\left\{\begin{array}{l}
\Lambda(t)=\sum L_{j}(t) \quad \text { with } \quad L_{j}(t)=4 \pi \lambda_{j} \int r^{2} \rho_{j}(r, t) \mathrm{d} r \\
S(t)=\sum S_{j}(t) \frac{L_{j}(t)}{\Lambda(t)}
\end{array}\right.
$$

where the index $j$ stands for all positron states: $p$-Ps, free $\mathrm{e}^{+}$, and one or more $o$-Ps states. Generally the $S_{j}(t)$ coefficients may be function of the positron age $t$. However, if we assume that the Ps is formed in its final state, particularly that the trapping of Ps into the free-volume sites is immediate, then each contribution has a characteristic constant $S_{j}$ value.

A non-linear least square computer programme was written to fit Eq. (3.2) to the experimental data and the population densities $\rho_{i}$ are obtained by numerical integration of (3.1). Because in Eq. (3.2) there is an integration over the spatial coordinate, the fitting cannot yield information on the absolute values of the blob sizes $\sigma_{\mathrm{b}}$ and $\sigma_{+}$. Therefore we define the electron blob size $\sigma_{\mathrm{b}}$ as the unity of size, which means that all space-dependent parameters are rescaled. The non-linear fitting parameters are thus $\left[\tau_{\mathrm{b}}, \sigma_{+}^{*}=\sigma_{+} / \sigma_{\mathrm{b}}, D_{+}^{*}=D_{+} / \sigma_{\mathrm{b}}^{2}, \kappa_{i}^{*}=\kappa_{i} / \sigma_{\mathrm{b}}^{3}\right]$ and the respective decay constants $\lambda_{i}$ of the different positron states. The $S_{j}$-values are linear parameters in the fitting procedure.

For the analysis of the PMMA data it was necessary to split the $o$-Ps contribution into 2 parts, i.e. (3.1) was extended into 5 equations. The longest-lived $o$-Ps component $\tau_{o-P s}^{(2)}$ corresponds to Ps trapped into the free-volume sites. The meaning of the shorter one $\tau_{o-P s}^{(1)}$ is still uncertain. It may be due to the statistical splitting-up of a distribution of hole sizes [6], or to a Ps attached to a molecule of the polymer. Details of the fitting procedure were given by $[1,5]$. 


\section{Results and discussion}

\subsection{AMOC on PMMA}

Figure 1a shows the experimental and fitted $S(t)$ curves for linear PMMA at two temperatures. We obtained excellent fittings by assuming that the hole formation rate $\kappa \rho_{\mathrm{h}}$ is negligible. This suggests that the hole model for formation of Ps is only of second order importance to the blob mechanism. The obtained relative contributions $L_{j}(t) / \Lambda(t)$ are presented in Fig. 1b for the first 2 nanoseconds. One sees that at $t=0$ only free positron decay is present and that the only contribution to the $S(t)$ curve will be $S_{+}$. There is a fast build-up of the contribution of para-positronium $S_{p-\mathrm{Ps}}$, which reaches its maximum after about $0.05 \mathrm{~ns}$, with a corresponding sharp decrease in the free positron contribution. As $S_{p-\mathrm{Ps}}>S_{+}$, this will lead to an increase in $S(t)$ during the first $50 \mathrm{ps}$. This building-up of the $p$-Ps contribution is characteristic of a delayed formation of Ps which is an
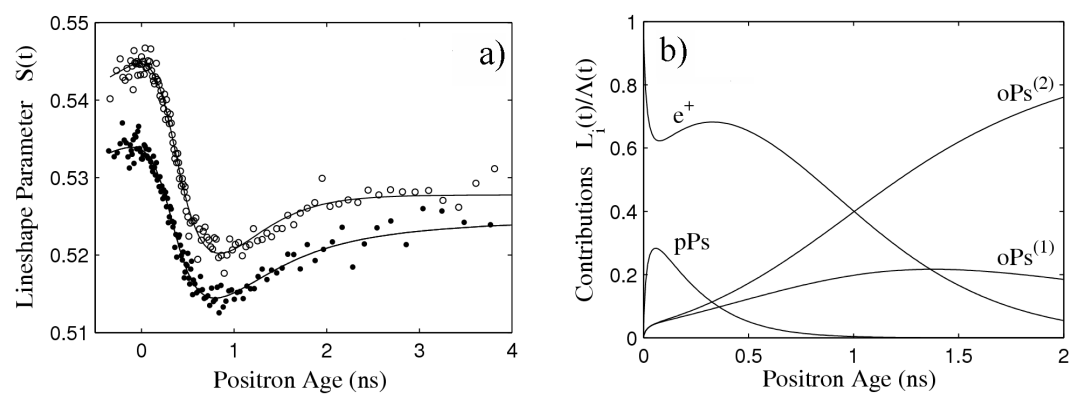

Fig. 1. Results of blob fitting of AMOC on PMMA. (a) Experimental $S(t)$ data of linear PMMA at $420 \mathrm{~K}$ (upper curve) and at $100 \mathrm{~K}$ (lower curve). The solid lines are the best fits obtained with the blob model. (b) Reconstruction of the relative contributions to the lifetime spectrum for linear PMMA at $420 \mathrm{~K}$, before convolution with the time response function.

intrinsic consequence of the interaction of the positron with the blob. Thus an observed broadening of the line shape during the early few tens of picoseconds may be partly due to delayed Ps formation, and is not necessarily entirely due to the thermalization of epithermal $p$-Ps as is generally accepted in the work of the Stuttgart group [7, 8]. After $\approx 2$ ns the longest $o$-Ps contribution with $S_{o-\mathrm{Ps}}^{(2)}$ dominates the value of $S(t)$. Therefore the effects of delayed formation of Ps are observable only in the first $2 \mathrm{~ns}$, and they will not strongly interfere with the straightforward determination of the free volume hole size by a multi-component analysis. The total Ps fractions $I_{\mathrm{Ps}}^{(1,2)}$ are not fitted in the blob analysis, but they are obtained by integration of the different contributions, which depend on all other fitting parameters, but most strongly on the Ps formation rates $\kappa_{i} \rho_{i}$. The fitted parameters were reported in Table 1 of our former paper [1]. However, 
as said before, it is not possible to obtain absolute values for the blob sizes $\sigma_{\mathrm{b}}$ and $\sigma_{+}$. Therefore we can only state that $\sigma_{+}^{*}=\sigma_{+} / \sigma_{\mathrm{b}}$ is $\approx 1.17 \pm 0.15$, which is in agreement with the qualitative prediction of the model.

\subsection{Delayed formation of Ps}

In the analysis of PMMA we have considered Ps formation to take place only with the blob electrons, and found a young-age broadening due to delayed formation of Ps. Therefore the mean lifetime of positrons which form $p$-Ps is no longer the inverse of its decay constant

$$
\tau_{p-\mathrm{Ps}}=\frac{\int t L_{p-\mathrm{Ps}}(t) \mathrm{d} t}{\int L_{p-\mathrm{Ps}}(t) \mathrm{d} t} \neq \lambda_{p-\mathrm{Ps}}^{-1} \quad \text { with } \quad \lambda_{p-\mathrm{Ps}}=\eta \lambda_{p-\mathrm{Ps}}^{0}+\lambda_{\mathrm{po}}
$$

where $\eta$ is the contact density of the trapped $p$-Ps, $\lambda_{p-\mathrm{Ps}}^{0}=1 / 124 \mathrm{ps}^{-1}, \lambda_{\mathrm{po}}$ is the pick-off decay constant, and $\lambda_{p-\mathrm{Ps}}$ is the decay constant of the $p$-Ps, which is obtained from the fitting procedure. The contact density $\eta$ of confined Ps is lower than 1 which means that its intrinsic decay constant is also lower than its value in vacuum. With (4.1) we find $\tau_{p-\mathrm{Ps}}=155 \pm 5 \mathrm{ps}$ and $\tau_{p-\mathrm{Ps}}=160 \pm 5 \mathrm{ps}$ for the linear PMMA, respectively, at $100 \mathrm{~K}$ and at $420 \mathrm{~K}$. Extra positronium could be formed either with shallow trapped electrons [9] or by Ps-formation upon trapping of positrons in free-volume sites. Both processes are undistinguishable because they add identical terms in the summation of (3.1). Figure 2a shows the evolution of the mean lifetime of $p$-Ps based on the data of PMMA at $100 \mathrm{~K}$, with added formation of Ps. We see that $\tau_{p-\mathrm{Ps}}$ reaches surprisingly high values, although the decay constant $\lambda_{p-\text { Ps }}$ has a constant value only slightly smaller than $\lambda_{p-\mathrm{Ps}}^{0}$. This may be the reason why high values $\tau_{1}$ are generally found in a classical three component analysis of polymers. Figure $2 \mathrm{~b}$ shows the simulated evolution of the total fraction of positronium $I_{\mathrm{Ps}}$ with increasing density of shallow trapped electrons or free-volume sites.
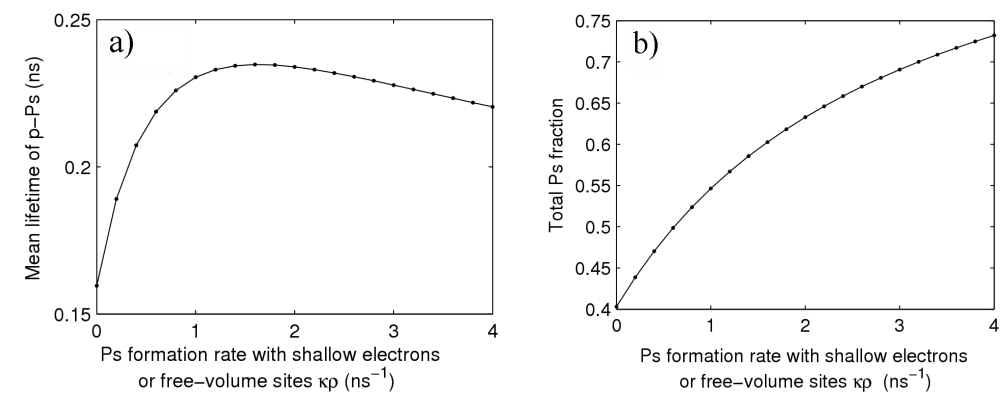

Fig. 2. Effect of free-volume sites or of shallow trapped electrons. The reference parameters at $\kappa \rho=0$ correspond to PMMA at $100 \mathrm{~K}$ : (a) evolution of the mean lifetime of $p$-Ps; (b) evolution of the total intensity of Ps. 


\section{Conclusions}

As a conclusion, we have shown that the blob model for positronium formation gives an adequate interpretation of AMOC data recorded in linear and cross-linked PMMA at two temperatures. It describes delayed formation of positronium and explains the young age broadening. The intensity of the $o$-Ps contribution is a complicated function of the blob chemistry. Shallow trapped electrons and free volume sites add extra channels for delayed Ps formation.

\section{Acknowledgments}

The authors thank Dr. H. Stoll, Prof. D. Carstanjen and the Max-Planck-Institute for Metal Research (Stuttgart, Germany) for the use of their MeV positron beam, the Fund for Scientific Research - Flanders (Belgium) (F.W.O. Vlaanderen), and the Ghent University research fund, for financial support. This work is also supported by the IAP program P5/01 of the Belgian Science Policy Office.

\section{References}

[1] C. Dauwe, B. Van Waeyenberge, J. De Baerdemaeker, Mater. Sci. Forum 445446, 229 (2004).

[2] V.M. Byakov, S.V. Stepanov, J. Radioan. Nucl. Chem. 210, 371 (1996).

[3] S.V. Stepanov, V.M. Byakov, Principles and Applications of Positron and Positronium Chemistry, Eds. Y.C. Jean, P.E. Mallon, D.M. Schrader, World Scientific, Singapore 2003, p. 117.

[4] H. Stoll, M. Koch, K. Maier, J. Major, Nucl. Instrum. Methods Phys. Res. B 56, 582 (1991).

[5] C. Dauwe, B. Van Waeyenberge, N. Balcaen, Phys. Rev. B 68, 132202 (2003).

[6] G. Dlubek, Ch. Hübner, S. Eichler, Nucl. Instrum. Methods Phys. Res. B 142 , 191 (1998).

[7] H. Stoll, P. Castellaz, A. Siegle, in Ref. [3], p. 362.

[8] H. Stoll, P. Castellaz, S. Koch, J. Major, H. Schneider, A. Seeger, Mater. Sci. Forum 255-257, 92 (1997).

[9] T. Hirade, Mater. Sci. Forum 445-446, 234 (2004). 